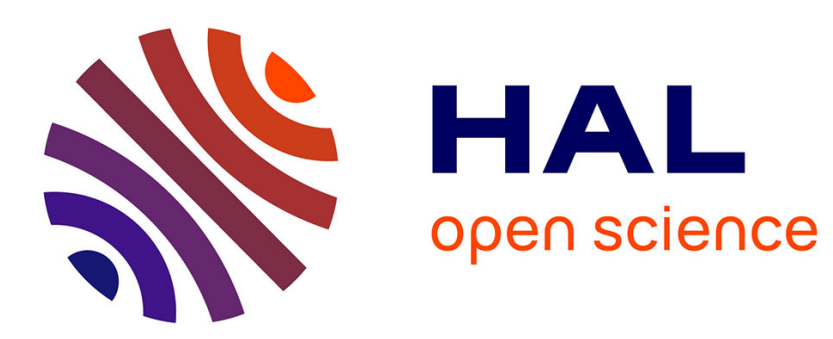

\title{
Une méthode de mesure simple et directe du facteur de bruit pour les amplificateurs et les transistors
}

\author{
G.A. Blasquez
}

\section{To cite this version:}

G.A. Blasquez. Une méthode de mesure simple et directe du facteur de bruit pour les amplificateurs et les transistors. Revue de Physique Appliquée, 1980, 15 (5), pp.1007-1012. 10.1051/rphysap:019800015050100700 . jpa-00244806

\section{HAL Id: jpa-00244806 https://hal.science/jpa-00244806}

Submitted on 1 Jan 1980

HAL is a multi-disciplinary open access archive for the deposit and dissemination of scientific research documents, whether they are published or not. The documents may come from teaching and research institutions in France or abroad, or from public or private research centers.
L'archive ouverte pluridisciplinaire HAL, est destinée au dépôt et à la diffusion de documents scientifiques de niveau recherche, publiés ou non, émanant des établissements d'enseignement et de recherche français ou étrangers, des laboratoires publics ou privés. 


\title{
Une méthode de mesure simple et directe du facteur de bruit pour les amplificateurs et les transistors
}

\author{
G. A. Blasquez \\ Laboratoire d'Automatique et d'Analyse des Systèmes, \\ 7, avenue du Colonel-Roche, 31400 Toulouse, France \\ (Reçu le 29 octobre 1979, révisé le 22 janvier 1980, accepté le 28 janvier 1980)
}

\begin{abstract}
Résumé. - En connectant la résistance de source entre l'entrée et la sortie de quadripôles comme les amplificateurs intégrés, les transistors à effet de champ et les transistors bipolaires, le facteur de bruit peut être déterminé directement à partir d'une seule mesure de bruit. Pour les amplificateurs, la méthode est utilisable tant que le gain en tension est égal ou supérieur à cinq et dans un intervalle de valeurs de résistances de source inversement proportionnel au cube de la fréquence. Pour les transistors, elle est applicable tant que le gain en courant en régime de court-circuit à la sortie est approximativement supérieur ou égal à cinq et dans un intervalle de valeurs de résistances de source inversement proportionnel à la fréquence. Cette méthode est plus simple à mettre en œuvre, plus précise et plus rapide que les méthodes classiques.
\end{abstract}

Abstract. - By connecting the source resistance between the input and output of a two-port such as integrated amplifiers, field effect transistors and bipolar transistors, the noise figure can be directly determined from a single noise measurement. In the case of amplifiers, the method can be used as long as the voltage gain is equal or greater than five and over a source resistance range which is inversely proportional to the cube of the frequency. For transistors it is applicable when the short circuit current gain is equal or greater than five and over a source resistance range which is inversely proportional to the frequency. This method is simpler to use, is more accurate and is faster than classical methods.

1. Introduction. - La mesure du facteur de bruit $F$ des amplificateurs intégrés, des transistors bipolaires et des transistors à effet de champ est une opération fréquemment effectuée par les fabricants et les utilisateurs de ces composants. Les méthodes actuelles étant relativement délicates à mettre en œuvre, peu précises et peu rapides, il est souhaitable d'en rechercher de nouvelles qui ne présenteraient pas ces inconvénients.

Dans une note récente [1], il a été montré que dans le domaine des basses et moyennes fréquences, $F$ peut être obtenu à partir d'une mesure directe du bruit ramené à l'entrée de l'étage constitué par le composant à caractériser et ses résistances de source et de charge. Dans cette méthode, l'étage est inséré dans un circuit réactionné qui se comporte comme un système à boucle unitaire. Le bruit à la sortie du circuit est alors égal au bruit ramené à l'entrée de l'étage et sa détermination est immédiate. A priori, cette technique semble présenter toutes les propriétés recherchées et une réflexion approfondie mérite d'être effectuée pour en préciser les performances potentielles et les limitations.
L'étude qui suit peut être considérée comme une première contribution à cette réflexion. Elle porte sur la classe des quadripôles amplificateurs, comme les transistors et les amplificateurs opérationnels. Dans ce cas très important en pratique, on monte sans difficultés des étages qui ont un gain en tension notable. Par suite, l'emploi d'un amplificateur de mesure (voir [1]) qui donne à l'asservissement un gain en boucle ouverte suffisant, devient superflu. Sa suppression présente deux avantages : d'une part la conception, la réalisation et la mise au point du circuit sont immédiates, d'autre part les limitations fréquentielles résultant de l'utilisation de cet amplificateur n'existent plus. Le montage fonctionne alors dans un domaine de fréquence étendu.

\section{Les objectifs de l'étude sont :}

a) de rappeler le principe de la détermination directe du facteur de bruit ;

b) de donner la structure du circuit correspondant pour les quadripôles amplificateurs;

c) d'effectuer la synthèse de ce circuit et d'en établir les limitations intrinsèques ; 
d) d'appliquer les résultats généraux obtenus en $c$ ) aux amplificateurs et transistors.

L'article est divisé en deux parties essentielles. Dans la première sont regroupés les aspects abstraits. Dans la deuxième sont traitées les applications. Le cas des transistors qui présente de nombreuses analogies avec celui des amplificateurs est reporté en annexe. Sous cette forme, l'étude donne, d'une manière concise, des résultats pratiques directement utilisables par les nombreux chercheurs, ingénieurs et techniciens qui sont confrontés au problème délicat des mesures de bruit.

2. Etude théorique. - 2.1 PrINCIPE. - Soit $Q$ un quadripôle bruyant quelconque. Le comportement en bruit de fond de ce quadripôle est équivalent à celui d'un quadripôle non bruyant associé à deux générateurs de bruit placés à l'entrée [2]. Désignons par $E_{n \mathrm{Q}}$ et $I_{n \mathrm{Q}}$ ces deux générateurs. Le facteur de bruit du quạdripôle alimenté par une source dont le bruit propre est égal à $\left\langle\left|E_{n \mathrm{G}}\right|^{2}\right\rangle$ et dont la résistance interne est égale à $R_{\mathrm{G}}$, peut s'écrire [2] :

$$
F=\frac{\left\langle\left|E_{n \mathrm{G}}\right|^{2}\right\rangle+\left\langle\left|E_{n \mathrm{Q}}+R_{\mathrm{G}} I_{n \mathrm{Q}}\right|^{2}\right\rangle}{\left\langle\left|E_{n \mathrm{G}}\right|^{2}\right\rangle} .
$$

Par hypothèse le bruit de la source est thermique et sa valeur quadratique moyenne est donnée par la formule de Nyquist-Johnson.

Il découle de (1) que le facteụr de bruit peut être simplement et rapidement déterminé si le numérateur peut être mesuré d'une manière directe.

\subsection{Structure et ANAlyse DU CirCUit DE baSE. -} Le circuit de base est représenté sur la figure 1. La boîte noire portant la lettre Q est un quadripôle quelconque. En général l'entrée et la sortie du quadripôle sont connectées à des circuits' de polarisation et de charge qui sont simulés sur la figure 1 par les dipôles B et $\mathrm{L}$. De plus un dipôle $\mathrm{S}$ est connecté entre l'entrée et la sortie de $\mathrm{Q}$.

Dans ce qui suit on ne s'intéresse qu'au régime dynamique petits signaux où l'on peut considérer que le circuit de base se comporte comme un système linéaire. Pour simplifier on admettra que les dipôles $\mathrm{B}, \mathrm{L}$ et $\mathrm{S}$ sont purement résistifs. Soient $G_{\mathrm{B}}$ et $G_{\mathrm{L}}$ les conductances respectives des dipôles $\mathrm{B}$ et $\mathrm{L}$ et soit $R_{\mathrm{S}}$ la résistance du dipôle $\mathrm{S}$. Nous désignerons par $y_{11}, y_{12}$, $y_{21}$ et $y_{22}$ les éléments de la matrice $Y_{\mathbf{Q}}$ du quadripôle Q.

D'une manière générale, tous les éléments du circuit

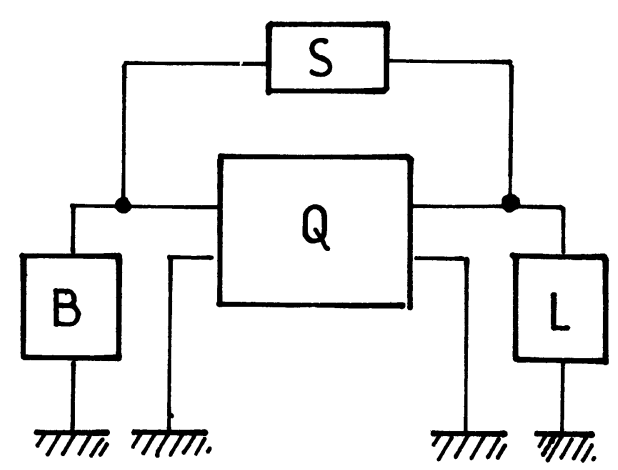

Fig. 1. - Schéma block du circuit de base.

[Block diagram of the basic circuit.]

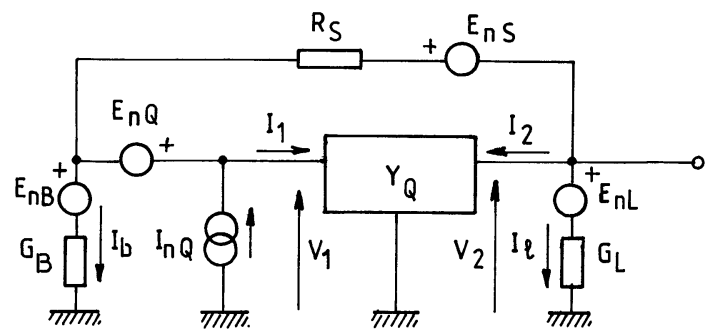

Fig. 2. - Circuit équivalent en bruit de fond.

[Noise equivalent circuit.]

de base sont bruyants. Conformément à l'usage courant on peut simuler le bruit de chaque dipôle par un dipôle parfait (sans bruit) associé à un générateur de bruit en tension placé en série [3]. Soient $E_{n S}$, $E_{n \mathrm{~L}}$ et $E_{n \mathrm{~B}}$ les générateurs de bruit en tension associés respectivement aux dipôles $S$, $L$ et $B$. Comme en section 2.1 on désignera par $E_{n \mathrm{Q}}$ et $I_{n \mathrm{Q}}$ les générateurs de bruit équivalents au bruit du quadripôle $\mathrm{Q}$.

Le circuit équivalent en bruit de fond au circuit de base est représenté sur la figure 2. Les courants et les tensions qui sont indiqués sur cette figure sont dus aux générateurs de bruit.

L'application des règles de Kirchhoff au circuit de la figure 2 donne :

$$
\begin{aligned}
& I_{1}=y_{11} V_{1}+y_{12} V_{2} \\
& I_{2}=y_{21} V_{1}+y_{22} V_{2} \\
& V_{2}=E_{n \mathrm{~L}}+I_{l} G_{\mathrm{L}}^{-1} \\
& V_{2}=V_{1}-E_{n \mathrm{Q}}-R_{\mathrm{S}}\left(I_{2}+I_{l}\right)-E_{n \mathrm{~S}} \\
& I_{n \mathrm{Q}}=I_{1}+I_{2}+I_{l}+I_{\mathrm{b}} \\
& V_{1}=E_{n \mathrm{Q}}+E_{n \mathrm{~B}}+I_{\mathrm{b}} G_{\mathrm{B}}^{-1} .
\end{aligned}
$$

La résolution du système (2) à (7) aboutit à une expression de la forme :

$$
V_{2}=k_{1}\left[k_{2} E_{n \mathrm{~S}}+\left(k_{3} E_{n \mathrm{Q}}+I_{n \mathrm{Q}}\right)+I_{n \mathrm{~B}}+k_{4} I_{n \mathrm{~L}}\right]
$$


avec $: I_{n \mathrm{~B}}=G_{\mathrm{B}} E_{n \mathrm{~B}}, I_{n \mathrm{~L}}=G_{\mathrm{L}} E_{n \mathrm{~L}}$,

$$
\begin{aligned}
& k_{1}=\frac{1-R_{\mathrm{S}} y_{21}}{y_{11}+y_{12}+y_{21}+y_{22}+G_{\mathrm{L}}+G_{\mathrm{B}}+R_{\mathrm{S}}\left(y_{11} y_{22}-y_{12} y_{21}+y_{22} G_{\mathrm{B}}+y_{11} G_{\mathrm{L}}+G_{\mathrm{B}} G_{\mathrm{L}}\right)} \\
& k_{2}=-\frac{y_{11}+y_{21}+G_{\mathrm{B}}}{1-R_{\mathrm{S}} y_{21}} \\
& k_{3}=-\frac{y_{11}+y_{21}+R_{\mathrm{S}} y_{21} G_{\mathrm{B}}}{1-R_{\mathrm{S}} y_{21}} \\
& k_{4}=\frac{1+R_{\mathrm{S}}\left(y_{11}+G_{\mathrm{B}}\right)}{1-R_{\mathrm{S}} y_{21}} .
\end{aligned}
$$

La valeur quadratique moyenne de la tension de sortie est égale à :

$$
\left\langle\left|V_{2}\right|^{2}\right\rangle=\left|k_{1}\right|^{2}\left\{\left|k_{2}\right|^{2}\left\langle\left|E_{n \mathrm{~S}}\right|^{2}\right\rangle+\left\langle\left|k_{3} E_{n \mathrm{Q}}+I_{n \mathrm{Q}}\right|^{2}\right\rangle+\left\langle\left|I_{n \mathrm{~B}}\right|^{2}\right\rangle+\left|k_{4}\right|^{2}\left\langle\left|I_{n \mathrm{~L}}\right|^{2}\right\rangle\right\} .
$$

Dans l'établissement de (13) à partir de (8) il est tenu compte du fait que $I_{n \mathrm{~L}}, I_{n \mathrm{~B}}$ et $E_{n \mathrm{~S}}$ ayant des origines physiques distinctes ne sont ni corrélés entre eux ni corrélés avec $E_{n \mathrm{Q}}$ ou $I_{n \mathrm{Q}}$.

2.3 CARACTÉRISTIQUES DU CIRCUIT DE DÉTERMINATION DIRECTE DU FACTEUR DE BRUIT. - D'après 2.1, pour que le circuit de la figure 1 permette la détermination directe de $F$, il faut que $\left\langle\left|V_{2}\right|^{2}\right\rangle$ ait la forme du numérateur de (1), i.e. :

$$
\left\langle\left|V_{2}\right|^{2}\right\rangle \approx\left\langle\left|E_{n \mathrm{~S}}\right|^{2}\right\rangle+\left\langle\left|E_{n \mathrm{Q}}+R_{\mathrm{S}} I_{n \mathrm{Q}}\right|^{2}\right\rangle
$$

Dans ce cas, $F$ est donné par le rapport $\left\langle\left|V_{2}\right|^{2}\right\rangle /\left\langle\left|E_{n \mathrm{~S}}\right|^{2}\right\rangle$. L'expression (14) implique alors que le circuit de base ait les caractéristiques suivantes :

$$
\begin{gathered}
\left|k_{1}\right| \approx R_{\mathrm{S}} \\
\left|k_{2}\right| \approx R_{\mathrm{S}}^{-1} \\
k_{3} \approx R_{\mathrm{S}}^{-1} \\
\left.\left(\left|k_{2}\right|^{2}\left\langle\left|E_{n \mathrm{~S}}\right|^{2}\right\rangle+\left\langle\left|I_{n \mathrm{Q}}+k_{3} E_{n \mathrm{Q}}\right|^{2}\right\rangle\right)\right\rangle\left(\left\langle\left|I_{n \mathrm{~B}}\right|^{2}\right\rangle+\left|k_{4}\right|^{2}\left\langle\left|I_{n \mathrm{~L}}\right|^{2}\right\rangle\right) .
\end{gathered}
$$

L'inégalité (18) signifie que les contributions en bruit dues au circuit de charge et au circuit de polarisation doivent être négligeables par rapport au bruit produit par le quadripôle et la résistance $R_{\mathrm{s}}$. C'est une contrainte générale de tous les circuits de mesure du facteur de bruit quelle que soit la méthode employée. En explicitant $\left\langle\left|I_{n \mathrm{~B}}\right|^{2}\right\rangle$ et $\left\langle\left|I_{n \mathrm{~L}}\right|^{2}\right\rangle$ à l'aide de la formule de Nyquist-Johnson, on peut montrer que pour un quadripôle et une résistance $R_{\mathrm{S}}$ quelconques, (18) stipule que $G_{\mathrm{B}}$ et $G_{\mathrm{L}}$ doivent être aussi faibles que possible. On précisera par la suite la signification exacte de « aussi faibles que possible ».

Avant de poursuivre l'analyse de la signification de (15) à (18), il est intéressant de noter que la résistance de contre-réaction $R_{\mathrm{S}}$ joue le rôle dans (14) d'une résistance de source. En conséquence nous utiliserons dans ce qui suit l'un ou l'autre de ces termes pour désigner cette résistance. de :

En explicitant (15) à (17) à l'aide de (9) à (11) on montre sans difficultés que (15) à (17) sont la conséquence

$$
\begin{gathered}
\left|1-R_{\mathrm{S}} y_{21}\right| \approx R_{\mathrm{S}}\left|y_{21}\right| \\
\left|y_{11}+y_{12}+y_{21}+y_{22}+G_{\mathrm{B}}+G_{\mathrm{L}}+R_{\mathrm{S}}\left(y_{11} y_{22}-y_{21} y_{12}+y_{22} G_{\mathrm{B}}+y_{11} G_{\mathrm{L}}+G_{\mathrm{B}} G_{\mathrm{L}}\right)\right| \approx\left|y_{21}\right| \\
y_{11}+y_{21}+R_{\mathrm{S}} y_{21} G_{\mathrm{B}} \approx y_{21} .
\end{gathered}
$$

Il découle de (19) que le circuit de base ne permet pas la détermination directe du facteur de bruit pour les résistances de source très faibles.

La relation (20) contient beaucoup de paramètres. L'examen de l'influence de chacun d'eux conduirait à une discussion trop détaillée qui dépasserait le cadre limité d'un article. Néanmoins une analyse succincte de (20) indique en premier lieu que le quadripôle à caractériser doit avoir un paramètre $y_{21}$ nettement supérieur à $y_{11}$, $y_{12}$ et $y_{22}$. Dans leur domaine nominal de fonctionnement, la condition précédente est très souvent vérifiée pour les transistors bipolaires, les transistors à effet de champ et les amplificateurs intégrés. Elle n'est donc que rarement restrictive et dans ce qui suit on admettra qu'elle est toujours vérifiée. Pour simplifier on admettra de plus que les circuits de polarisation et de charge sont bien conçus c'est-à-dire que $G_{\mathrm{B}}$ et $G_{\mathrm{L}}$ sont très petits conformé- 
ment à une remarque effectuée lors de l'examen de (18). Dans ce cas les termes de (20) qui contiennent au moins un de ces paramètres peuvent être négligés et l'expression se réduit à :

$$
\left|y_{21}+R_{\mathrm{S}}\left(y_{11} y_{22}-y_{21} y_{12}\right)\right| \approx\left|y_{21}\right| \text {. }
$$

Il découle de (22) que la résistance de source ne peut pas être supérieure à une valeur limite fonction des caractéristiques du quadripôle. Autrement dit, le circuit de la figure 1 ne permet pas la détermination directe du facteur pour les résistances de source très élevées. $\mathrm{Si}$, de plus, on fait l'hypothèse que l'un au moins des paramètres $y$ est une fonction de la fréquence, il découle de (22) que la valeur maximale admissible pour $R_{\mathrm{S}}$ dépendra ellemême de la fréquence. Ce résultat apparaîtra plus clairement dans la section 3. L'expression (21) définit une limitation assez simple pour la valeur de $G_{\mathbf{B}}$. En effet (21) implique que :

c'est-à-dire

$$
y_{21}\left(1+R_{\mathrm{S}} G_{\mathrm{B}}\right) \approx y_{21}
$$

$$
G_{\mathrm{B}} \ll R_{\mathrm{S}}^{-1} \text {. }
$$

Notons que quand cette condition est réalisée, d'après la formule de Nyquist-Johnson on a :

$$
\left|k_{2}\right|^{2}\left\langle\left|E_{n \mathrm{~S}}\right|^{2}\right\rangle \gg\left\langle\left|I_{n \mathrm{~B}}\right|^{2}\right\rangle \text {. }
$$

Le choix de $G_{\mathrm{B}}$ à partir·de (23) concourt à réaliser (18).

Nous pouvons maintenant préciser l'intervalle des valeurs à donner à $G_{\mathrm{L}}$. Il découle de (20) et des hypothèses effectuées précédemment que :

$$
G_{\mathrm{L}} \ll \frac{\left|y_{21}\right|}{\left|1+R_{\mathrm{S}} y_{11}\right|} .
$$

De plus quand (23), (15), (16), (17) et (19) sont réalisés, l'expression (18) implique :

$$
\left.\left(\left\langle\left|E_{n \mathrm{~S}}\right|^{2}\right\rangle+\left\langle\left|E_{n \mathrm{Q}}+R_{\mathrm{S}} I_{n \mathrm{Q}}\right|^{2}\right\rangle\right)\right\rangle\left|\frac{1+R_{\mathrm{S}}\left(y_{11}+G_{\mathrm{B}}\right)}{y_{21}}\right|^{2}\left\langle\left|I_{n \mathrm{~L}}\right|^{2}\right\rangle .
$$

En explicitant $\left\langle\left|I_{n \mathrm{~L}}\right|^{2}\right\rangle$ à l'aide de la formule de Nyquist-Johnson, (26) peut se mettre sous la forme :

$$
G_{\mathrm{L}} \ll \frac{1}{4 k T \Delta f}\left|\frac{y_{21}}{1+R_{\mathrm{S}}\left(y_{11}+G_{\mathrm{B}}\right)}\right|^{2}\left(\left\langle\left|E_{n \mathrm{~S}}\right|^{2}\right\rangle+\left\langle\left|E_{n \mathrm{Q}}+R_{\mathrm{S}} I_{n \mathrm{Q}}\right|^{2}\right\rangle\right)
$$

où $k$ désigne la constante de Boltzmann, $T$ la température et $\Delta f$ un intervalle élémentaire de fréquences. Quand les caractéristiques de bruit du quadripôle sont totalement inconnues, on peut estimer un majorant de $G_{\mathrm{L}}$ en ne tenant pas compte des termes correspondants dans (27). Dans ce cas, en explicitant $\left\langle\left|E_{n \mathrm{~S}}\right|^{2}\right\rangle$ on peut remplacer (27) par :

$$
G_{\mathrm{L}}<R_{\mathrm{S}}\left|\frac{y_{21}}{1+R_{\mathrm{S}}\left(y_{11}+G_{\mathrm{B}}\right)}\right|^{2} .
$$

En pratique, pour définir $G_{\mathrm{L}}$ il faut donc estimer (25) et (28) et ne retenir que la condition la plus contraignante.

3. Applications. - Dans ce qui suit, les conclusions générales de la section 2 sont appliquées aux cas des amplificateurs et des transistors. Néanmoins, comme l'analyse à effectuer est la même dans chaque cas, elle ne sera pas répétée pour les transistors et les résultats correspondants seront donnés en annexe.

Considérons un amplificateur de tension dont les caractéristiques représentent typiquement celles des amplificateurs intégrés :

- gain en tension

$$
A_{\mathrm{v}}(\omega)=A_{\mathrm{vo}} /\left(1+j \omega / \omega_{\mathrm{c}}\right)=10^{5} /(1+j \omega / 20 \pi),
$$

- impédance d'entrée

$$
z_{\mathrm{i}}=r_{\mathrm{i}} /\left(1+j \omega / \omega_{\mathrm{i}}\right)=10^{7} /\left[1+j \omega /\left(3,3 \times 10^{4}\right)\right]
$$

$\Omega$, résistance de sortie $r_{0}=10^{3} \Omega$, admittance de transfert $y_{12} \approx 0$ mho.

En fonction de ces données, les paramètres de l'amplificateur $Q$ s'écrivent :

$$
y_{11}=z_{i}^{-1}, \quad y_{21}=-A_{\mathrm{V}}(\omega) / r_{0}, \quad y_{22}=r_{0}^{-1} .
$$

Le courant continu de polarisation peut être fourni 
à l'entrée à travers $R_{\mathrm{S}}$ et par suite le circuit de polarisation est inutile, $G_{\mathrm{B}}=0$ mho.

Notons également qu'il est légitime d'admettre que $A_{\mathrm{V}}$ est purement imaginaire c'est-à-dire que nous pouvons écrire les approximations :

$$
A_{\mathrm{v}} \approx A_{\mathrm{v} 0} \frac{\omega_{\mathrm{c}}}{j \omega} \quad \text { et } \quad y_{21} \approx j \frac{A_{\mathrm{v} 0} \omega_{\mathrm{c}}}{r_{0} \omega} .
$$

Cette approximation revient à ne considérer que des fréquences supérieures à $10 \mathrm{~Hz}$ dans l'exemple numérique choisi. Etablissons en premier lieu le domaine des fréquences où la méthode décrite en section 2 est applicable. On a vu que la méthode est applicable si $y_{22}^{2} \ll\left|y_{21}\right|^{2}$. En bornant le domaine des fréquences à $\omega_{\mathrm{M}}=\frac{\omega_{\mathrm{c}} A_{\mathrm{v} 0}}{5}, y_{22}^{2}$ n'excède pas $4 \%$ de $\left|y_{21}\right|^{2}$.

En pratique, la précision courante des mesures de facteur de bruit n'atteint pas $10 \%$. On peut donc considérer que la méthode est applicable jusqu'à $\omega=\omega_{\mathrm{M}}$. Pour l'exemple choisi, la limite se situera vers $200 \mathrm{kHz}$ alors que l'amplificateur sera utilisable jusqu'à $1 \mathrm{MHz}$ environ.

Déterminons maintenant la plage des valeurs admissibles pour $R_{\mathrm{s}}$. A partir des données précédentes et de (19) on établit que $R_{\mathrm{S}} \gg \mathrm{r}_{0} \frac{\omega}{A_{\mathrm{v} 0} \omega_{\mathrm{c}}}$. En particulier, si $\omega=\omega_{\mathrm{M}}, R_{\mathrm{S}} \gg \frac{r}{5}$, soit pour l'exemple choisi $R_{\mathrm{S}} \gg 200 \Omega$. Pour déterminer la borne supérieure des valeurs admissibles pour $R_{\mathrm{S}}$ il est intéressant de remarquer de plus que pour la plage des fréquences considérées,

$$
\operatorname{Re}\left(y_{21}\right)>\operatorname{Re}\left(y_{11}\right) \text { et } \operatorname{Im}\left(y_{21}\right)>\operatorname{Im}\left(y_{11}\right) \text {. }
$$

Si nous négligeons en outre les termes contenant $G_{\mathrm{L}}$ (nous justifierons ci-desșous cette hypothèse), l'expression (20) se réduit à :

$$
\left|y_{21}+R_{\mathrm{S}} y_{22} y_{11}\right| \approx\left|y_{21}\right| \text {. }
$$

Pour que cette condition soit vérifiée, il faut et il suffit qu'elle soit satisfaite quand $\left|y_{21}\right|$ est minimum. Ce minimum est obtenu pour $\omega=\omega_{\mathrm{M}}$ il est égal à $\left|y_{21}\left(\omega=\omega_{M}\right)\right|=5 / r_{0}$. Pour cette fréquence la partie imaginaire de $y_{11}$ est prépondérante et l'on peut considérer que tous les termes de (29) sont imaginaires purs. L'expression (29) s'écrit alors :

$$
\left|\frac{A_{\mathrm{vo}} \omega_{\mathrm{c}}}{r_{0} \omega}+\frac{R_{\mathrm{S}} \omega}{r_{0} r_{i} \omega_{i}}\right| \approx\left|\frac{A_{\mathrm{v} 0} \omega_{\mathrm{c}}}{r_{0} \omega}\right| .
$$

Elle est vérifiée si :

$$
R_{\mathrm{S}} \ll r_{i} A_{\mathrm{vo}} \omega_{i} \omega_{\mathrm{c}} / \omega^{2} .
$$

La valeur minimum de la limite est obtenue pour $\omega=\omega_{\mathrm{M}}$. Elle est égale à $25 r_{i} \omega_{i} / A_{\mathrm{vo}} \omega_{\mathrm{c}}$. Pour l'amplificateur choisi à titre d'exemple on a pour $f=200 \mathrm{kHz}$, $R_{\mathrm{S}} \ll 1,25 \mathrm{M} \Omega$.
Il découle de ce qui précède que la plage des valeurs admissibles pour $R_{\mathrm{S}}$ est inversement proportionnelle au cube de la plage des fréquences dans laquelle les mesures doivent être effectuées. En basse et moyenne fréquences, la détermination directe du facteur de bruit peut être effectuée pour la plupart des valeurs courantes de la résistance de source $R_{\mathrm{S}}$. Précisons maintenant les valeurs à donner à la conductance de charge $G_{\mathrm{L}}$. Elles doivent satisfaire simultanément (25) et (28). Cependant on remarque que le deuxième membre de (28) est égal au deuxième membre de (25) multiplié par le terme $R_{\mathrm{S}}\left|y_{21}\right| /\left|1+R_{\mathrm{S}} y_{11}\right|$. On note également que pour que (25) et (28) soient satisfaites il faut et il suffit qu'elles soient vérifiées quand leurs seconds nombres sont minimums. Ces minimums ont lieu quand $y_{21}$ est minimum et $y_{11}$ est maximum, c'est-à-dire, pour $\omega=\omega_{\mathrm{M}}$, comme nous l'avons déjà mentionné.

Nous avons vu aussi que (19) implique que

$$
R_{\mathrm{S}}\left|y_{21}\right| \gg 1 \text { et que }\left|y_{21}\right|>\left|y_{11}\right| \text {. }
$$

Il s'ensuit que le terme $R_{\mathrm{S}}\left|y_{21}\right| /\left|1+R_{\mathrm{S}} y_{11}\right|$ est supérieur à l'unité. La limite (28) est plus élevée que la limite donnée par (25). La limite (28) n'a pas à être prise en compte. En explicitant (25) pour $\omega=\omega_{\mathrm{M}}$, puis en effectuant les simplifications résultant de $R_{\mathrm{S}} \ll r_{i}$ et $A_{\mathrm{V} 0} \omega_{\mathrm{c}} \gg \omega_{i}$ on obtient sans difficultés que :

$$
G_{\mathrm{L}} \ll 5 / r_{0} .
$$

Pour l'exemple choisi une charge de l'ordre de $r_{0}$, c'est-à-dire égale à $1 \mathrm{k} \Omega$ environ, serait appropriée.

4. Conclusion. - En plaçant la résistance de source en contre-réaction entre l'entrée et la sortie d'un quadripôle on peut déterminer, sous certaines conditions, le facteur de bruit à partir d'une seule mesure de bruit. Pour que cette méthode s'applique correctement, il faut que le circuit constitué par le quadripôle, la résistance de contre-réaction, les circuits de polarisation et de charge ait les propriétés suivantes :

a) l'admittance $y_{21}$ du quadripôle soit prépondérante par rapport aux autres admittances du circuit, c'est-à-dire on doit étudier un bon amplificateur de gain notable,

b) les contributions en bruit des circuits de polarisation et de charge soient négligeables par rapport au bruit total du quadripôle et de la résistance de source et,

c) les valeurs de la résistance de source soient comprises entre certaines limites. Pour une valeur permise de cette résistance, la méthode s'applique sans problèmes, aux basses et moyennes fréquences, et sous certaines conditions, en hautes fréquences. Pour une fréquence de mesure fixée, la gamme permise des valeurs de la résistance de source est d'autant plus étendue que cette fréquence est faible.

La méthode est utilisable pour la caractérisation des amplificateurs de tension du type intégrés, les tran- 
sistors à effet de champ et les transistors bipolaires. Pour les amplificateurs de tension considérés, la méthode est applicable tant que le module du gain en tension est approximativement égal ou supérieur à cinq et pour une plage de valeurs de la résistance de source inversement proportionnelle au cube de la fréquence. Pour les transistors des deux types, le module du gain en courant en régime de sortie en court-circuit doit être approximativement égal ou supérieur à cinq tandis que l'étendue de la plage permise des valeurs de la résistance de source est inversement proportionnelle à la fréquence.

Pour comprendre d'une manière claire la portée pratique de la méthode proposée dans cet article, il faut se souvenir que les méthodes classiques de détermination $\mathrm{du}$ facteur de bruit impliquent la mesure du gain du quadripôle. A ce titre elles apparaissent comme des méthodes de mesure indirectes. $\mathrm{Au}$ contraire la méthode proposée ne fait pas intervenir cette mesure et en conséquence il faut la considérer comme une méthode de mesure directe. Intrinsèquement elle est plus simple à mettre en œuvre, plus précise puisque les incertitudes provenant de la mesure du gain sont évitées, et enfin plus rapide.

Finalement les premiers essais de mise en application de la méthode donnent des résultats conformes à ceux présentés. Ils feront l'objet d'une publication complémentaire ultérieure.

\section{ANNEXE}

1. Cas des transistors à effet de champ. - Soient $g_{\mathrm{m}}$ la transconductance, $C_{\mathrm{gs}}$ la capacité grille source, $C_{\mathrm{gd}}$ la capacité grille drain et $g_{\mathrm{D}}$ la conductance de sortie. Les paramètres $y$ du transistor dans le montage source commune sont égaux à :

$$
\begin{array}{ll}
y_{11}=j \omega\left(C_{\mathrm{gs}}+C_{\mathrm{gd}}\right), & y_{12}=-j \omega C_{\mathrm{gd}}, \\
y_{21}=g_{\mathrm{m}}+j \omega C_{\mathrm{gd}}, & y_{22}=g_{\mathrm{D}}+j \omega C_{\mathrm{gd}} .
\end{array}
$$

En général $g_{\mathrm{m}} \gg g_{\mathrm{D}}, C_{\mathrm{gs}}>C_{\mathrm{gd}}$. A titre d'illustration on supposera :

$$
\begin{gathered}
g_{\mathrm{m}}=10^{-2} \mathrm{mho}, \quad g_{\mathrm{D}}=3 \times 10^{-6} \mathrm{mho}, \\
C_{\mathrm{gd}}=5 \mathrm{pF} \text { et } C_{\mathrm{gs}}=10 \mathrm{pF} .
\end{gathered}
$$

Pour les fréquences telles que $\omega<g_{\mathrm{m}} /\left(2 C_{\mathrm{gd}}+C_{\mathrm{gs}}\right)$, on a

$$
\begin{gathered}
y_{21} \approx g_{\mathrm{m}}, \quad y_{21}>\left|y_{11}\right|, \quad y_{21}>\left|y_{12}\right|, \\
y_{21}>\left|y_{22}\right| \text { et }\left|y_{21}+y_{12}+y_{22}+y_{11}\right| \approx y_{21} .
\end{gathered}
$$

Dans ce cas, il découle de (19) et (22) que.

$$
g_{\mathrm{m}}^{-1}<R_{\mathrm{S}}<\left(\omega C_{\mathrm{gd}}\right)^{-1}
$$

tandis que (23) impose $G_{\mathrm{B}} \ll \omega C_{\mathrm{gd}}$. Enfin en effectuant une analyse analogue à celle de la section 3 (25), donne $G_{\mathrm{L}}<g_{\mathrm{m}}$. Pour le transistor choisi à titre d'illustration, la méthode est applicable jusqu'à quelques dizaines de $\mathrm{MHz}$. La résistance de charge doit avoir une valeur de l'ordre de $1, \mathrm{k} \Omega$, tandis que la résistance de source doit être supérieure à $100 \Omega$. Enfin pour $f=16 \mathrm{kHz}, R_{\mathrm{s}}<2 \mathrm{M} \Omega$ et $R_{\mathrm{B}}=G_{\mathrm{B}}^{-1} \approx 20 \mathrm{M} \Omega$.

2. Cas des transistors bipolaires. - Soient $r_{\mathrm{e}}$ la résistance dynamique émetteur base dans le montage base commune, $C_{\mathrm{cb}}$ la capacité collecteur base, $h_{\mathrm{fe}}$ le gain en courant dans le montage émetteur commun, $\omega_{\mathrm{T}}$ la pulsation de transition. En première approximation le comportement des paramètres $y$ dans le montage émetteur commun peuvent être représentés par [4] :

$$
\begin{gathered}
y_{11} \approx\left(h_{\mathrm{fe}} r_{\mathrm{e}}\right)^{-1}, \quad y_{21} \approx r_{\mathrm{e}}^{-1}, \\
y_{12} \approx-j \omega C_{\mathrm{cb}} \text { et } y_{22} \approx-y_{12}
\end{gathered}
$$

avec

$$
h_{\mathrm{fe}}=h_{\mathrm{FE}} /\left(1+j \omega h_{\mathrm{FE}} / \omega_{\mathrm{T}}\right) .
$$

Pour illustrer ce cas nous considérerons un transistor ordinaire qui a les caractéristiques suivantes $h_{\mathrm{FE}}=100$, $f_{\mathrm{T}}=\omega_{\mathrm{T}} / 2 \pi=100 \mathrm{MHz}$ et $C_{\mathrm{cb}}=10 \mathrm{pF}$. Pour $\omega \leqslant \omega_{\mathrm{T}}$ on a d'une manière générale

$$
y_{21} \geqslant\left|y_{11}\right|, \quad y_{21}>\left|y_{12}\right| \text { et } y_{21}>\left|y_{22}\right| \text {. }
$$

Dans ce cas, (19) et (22) donnent $r_{\mathrm{e}}<R_{\mathrm{S}}<\left(C_{\mathrm{cb}}\right)^{-1}$. Pour les fréquences moyennes, la valeur de la résistance de source donnant le facteur de bruit minimum est :

$$
R_{\mathrm{S} 0} \approx\left[h_{\mathrm{FE}} r_{\mathrm{e}}\left(r_{\mathrm{e}}+R_{\mathrm{nb}}\right)\right]^{-1 / 2}
$$

où $R_{\mathrm{nb}}$ a une valeur de l'ordre de grandeur de la résistance de base [5]. La valeur de $R_{\mathrm{SO}}$ étant située à l'intérieur du domaine permis des variations de $R_{\mathrm{S}}$, le circuit de la figure 1 permet la détermination du facteur de bruit minimum. Par ailleurs, le report de la valeur maximale de $R_{\mathrm{S}}$ dans (23) donne $G_{\mathrm{B}} \ll C_{\mathrm{cb}} \omega$. Enfin pour des $R_{\mathrm{S}}$ faibles tels que $R_{\mathrm{S}}\left|y_{11}\right| \ll 1$, (25) donne $G_{\mathrm{L}}<r_{\mathrm{e}}^{-1}$ et pour des $R_{\mathrm{S}}$ élevés tels que

$$
R_{\mathrm{S}}\left|y_{11}\right| \gg 1, G_{\mathrm{L}}<h_{\mathrm{fe}} R_{\mathrm{S}}^{-1} .
$$

A $f=160 \mathrm{kHz}$ et $I_{\mathrm{c}}=1 \mathrm{~mA}, F$ peut être mesuré pour $26 \Omega<R_{\mathrm{S}}<10^{5} \Omega$ si $G_{\mathrm{B}} \approx 10^{-6}$ mho et $G_{\mathrm{L}} \approx 10^{-4}$ mho.

\section{Bibliographie}

[1] Blasquez, G., C.R. Hebd. Séan. Acad. Sci. B, sous presse.

[2] Haus, H. A., Proc. I.R.E., 1 (1960) 69.

[3] Grivet, P. et Blaquiere, A., Le Bruit de Fond (Masson) Paris, 1958.
[4] Lindmayer, J. and Wrigley, C., Fundamentals of Semiconductors Devices (Van Nostrand, Princeton), 1965.

[5] Blasquez, G., Caminade, J. and Legac, G., Physica 94B + C (1978) 359. 